\title{
Physics and modes of star cluster formation
}

\author{
Matthew R. Bate \\ School of Physics, University of Exeter, Stocker Road, Exeter EX4 4QL, UK \\ email: mbate@astro.ex.ac.uk
}

\begin{abstract}
I review the progress made in understanding the physics and modes of star cluster formation through the use of direct self-gravitating hydrodynamical simulations, including those that have recently been performed incorporating radiative transfer and magnetic fields.
\end{abstract}

Keywords. gravitation, hydrodynamics, MHD, radiative transfer, stellar dynamics, methods: numerical, stars: formation, stars: low-mass, brown dwarfs, stars: luminosity function, mass function

\section{Introduction}

Understanding star formation is a difficult problem. On the theoretical side, there is a huge range of scales involved (roughly 10 orders of magnitude in spatial scale and 12 orders of magnitude in temporal scale) and a large number of physical processes (gravity, supersonic turbulent fluid dynamics, magnetic fields, radiative transfer, chemistry). On the observational side, many stages of star formation (particularly massive star formation) are obscured and the entire process of star cluster formation takes millions of years, meaning that we need to try to piece together a complete theory using snapshots of the process taken from different regions.

Over the past decade, it has become possible to begin simulating the formation of clusters of stars directly using self-gravitating hydrodynamical calculations. This has opened up the possibility of trying to understand the origin of the statistical properties of stellar systems by conducting 'numerical experiments' in which the initial conditions and/or physical processes included are varied and the effects of these changes on the outputs of the star formation process (i.e., the statistical properties of the stellar systems) are measured.

In this contribution, I review the progress that has been made in simulating star cluster formation directly and what we have learnt to date of how the star-formation process depends on various physical processes and the initial conditions in molecular clouds.

\section{Hydrodynamical simulations}

The starting point for all direct simulations of star formation is self-gravitating compressible fluid dynamics. This is the minimum amount of physics required even to begin modelling the collapse and fragmentation of a molecular cloud to form a stellar cluster. However, even in this limited case, modelling the formation of a stellar cluster is beyond current computational resources because - as the collapse to form the first star ensues - the time steps required to evolve the calculation decrease by $\sim 10-12$ orders of magnitude. Thus, the calculation would grind to a halt as the first star forms. To get past this point, an approximation must be made. The standard procedure now used in both particle-based (Bate et al. 1995) and grid-based numerical methods (Krumholz et al. 2004 ) is to replace collapsing protostars by sink particles. Here, the dense gas within a specified radius of the centre of the protostar, the accretion radius, is combined into a 
single point mass with the same total mass and momentum as the gas it replaces. Gas that subsequently falls within this radius is accreted by the sink particle. This method allows the very short time steps that would be required to evolve the gas deeper inside the protostar to be avoided.

Klessen et al. (1998) used this method to follow the formation of a cluster of protostars from a cloud of molecular gas. Their calculation began with a static cloud in a periodic box with Gaussian density perturbations and produced 55 protostars following a roughly lognormal mass function. Subsequent calculations showed that the peak of the mass function was located near the mean Jeans mass of the initial clouds (Klessen \& Burkert 2001a,b). Klessen (2001) also investigated clouds with driven turbulence and found that the resulting stellar mass function was broader and flatter if the turbulence was driven on small scales than for large-scale driving. Offner et al. (2008) showed that there is not much difference between decaying turbulence and large-scale driving. Bonnell et al. (2003) performed a calculation of the collapse of an isolated $1000 \mathrm{M}_{\odot}$ molecular cloud containing decaying turbulence. This calculation pointed to the formation of large clusters occurring hierarchically, with the large stellar cluster containing more than 400 stars being formed through the merging of many subclusters.

A limitation of these initial models is that they only resolved the collapsing protostars to scales of several hundreds of AU (i.e., the sink particles had accretion radii of hundreds of $\mathrm{AU}$ ). Thus, they did not capture the opacity limit for fragmentation that occurs when collapsing gas becomes optically thick to its own radiation, and so did not capture all of the expected fragmentation (leading to incomplete mass functions), or resolve brown dwarfs, multiple systems and discs.

Bate et al. (2002a,b, 2003) performed the first cluster-formation calculation that resolved the opacity limit for fragmentation, thus capturing all fragmentation and resolving even the lowest-mass brown dwarfs. The calculation also resolved binaries with separations as close as $1 \mathrm{AU}$ and discs with radii down to $\approx 10 \mathrm{AU}$. The calculation demonstrated that star formation in clusters could be a higher chaotic and dynamical environment, with discs being truncated by dynamical encounters, fragmenting to form multiple systems, and stars and brown dwarfs being ejected from unstable multiple systems and escaping the cluster's gravitational potential. The calculation produced a modest 50 stars and brown dwarfs, enough for a crude comparison with observations. Within the uncertainties, the properties of the stars and brown dwarfs, such as the initial mass function (IMF), stellar multiplicity and the fraction of close binaries, were found to be in reasonable agreement with observations. As with the earlier, more poorly resolved, simulations, the stellar masses were found to originate through a process of competitive accretion, with the characteristic stellar mass being roughly the Jeans mass. All objects began with low masses and accreted from the cloud, typically to the mean thermal Jeans mass, but with a few stars in the centres of subclusters reaching higher masses and forming the high-mass end of the IMF. For those objects with final masses much lower than the typical Jeans mass (i.e., brown dwarfs), it was found that dynamical interactions and ejections from multiple systems were the key to their low masses (Bate et al. 2002a). They began with low masses (as did those objects that ended up with stellar masses), but their accretion was terminated when they were involved in dynamical interactions in small groups, which increased their velocities (typically to a few $\mathrm{km} \mathrm{s}^{-1}$ ) and ejected them from the dense cores in which they began forming. Thus, they were unable to accrete to the typical Jeans mass and ended up with substellar masses. Approximately three quarters of the brown dwarfs were found to originate from the fragmentation of massive circumstellar discs, while the remainder formed in dense filaments, fell into existing multiple systems and were ejected before they had accreted much mass. 
This first calculation of star cluster formation that resolved the opacity limit for fragmentation was followed by three similar calculations that investigated the dependence of stellar properties on the initial conditions in the molecular clouds and variations in the opacity limit for fragmentation. Bate \& Bonnell (2005) performed an identical calculation to that of Bate et al. (2003), but for a cloud with a smaller radius and nine times higher density. Thus, the mean thermal Jeans mass in the cloud was one third of that in the original calculation. The calculation produced a median stellar mass a factor of three lower than the original calculation, exactly matching the change in the mean Jeans mass. This confirmed the results of the earlier, more poorly resolved calculations, which indicated that the typical stellar mass was similar to the mean thermal Jeans mass. More recently, Jappsen et al. (2005) and Bonnell et al. (2006), using calculations with nonisothermal equations of state at very low molecular densities, showed that the transition from atomic-line to dust cooling could set an appropriate Jeans mass, which in turn produced the characteristic mass of the IMF.

Bate (2005) performed a third opacity-limited calculation, identical to that of Bate et al. (2003), except that the opacity limit for fragmentation was moved to a lower density by a factor of nine (i.e., the transition from an isothermal collapse to the gas becoming optically thick to its own radiation was assumed to occur earlier in the collapse). This increased the minimum mass by a factor of three to $\approx 9$ Jupiter masses. Such a change may occur, for example, in lower-metallicity gas which cools less effectively. Bate found that apart from increasing the minimum mass of a brown dwarf, this change to the equation of state produced no significant change in the rest of the IMF or the other stellar properties.

Finally, Bate (2009c) performed a fourth opacity-limited calculation, identical to that of Bate et al. (2003), except that the power spectrum of the initial turbulent velocity field was changed from $P(k) \propto k^{-4}$ (chosen to match the Larson (1981) scaling relations for velocity dispersion with length scale observed in molecular clouds) to $P(k) \propto k^{-6}$, which has more power on larger scales. The structures produced in the cloud during the evolution were very different to those found in the original calculation with large shocks and little small-scale structure. However, despite this difference, the stellar properties obtained were indistinguishable from those of the original calculation. In particular, the IMFs were almost identical.

Together, these four opacity-limited calculations show that the IMFs produced by hydrodynamical simulations using barotropic equations of state depend primarily on the mean thermal Jeans mass of the progenitor clouds and not on the exact value of the opacity limit for fragmentation or on the nature of the turbulent motions in the molecular gas. This is because, as long as there is sufficient structure in the gas and the equation of state allows fragmentation to produce many objects that interact dynamically, the processes of competitive accretion (Bonnell et al. 1997) and dynamical interactions and ejections (Bate et al. 2003) do not depend significantly on the initial structure and turbulent motions in the molecular cloud. Other properties, such as stellar multiplicity, were found not to vary significantly between any of the calculations for the same reason.

The calculations discussed above either did not resolve the opacity limit for fragmentation, and thus did not resolve low-mass objects or many multiple systems, or produced only a few dozen stars and brown dwarfs. Thus, making detailed comparisons with the observed properties of stars and brown dwarfs was not possible.

Recently, Bate (2009a) performed a hydrodynamical simulation of star cluster formation that resolved the opacity limit for fragmentation and produced well over 1000 stars and brown dwarfs. The calculation was identical to that of Bate et al. (2003), except that the cloud was an order of magnitude more massive $\left(500 \mathrm{M}_{\odot}\right)$. With so many 
objects, the statistical properties of low-mass stars and brown dwarfs are well determined and detailed comparison with observed stellar properties is possible. Bate found that many stellar properties were in good agreement with observations. For example, stellar multiplicity was found to be a strongly increasing function of primary mass with the multiplicity of very-low-mass objects $\left(0.03-0.10 \mathrm{M}_{\odot}\right), \mathrm{M}$ dwarfs, and solar-type stars increasing from $\approx 20$ to $60 \%$, as observed. The trends for low-mass binaries to have smaller separations and equal-mass components were also reproduced, and even the distribution of relative orientations of the orbital planes of triple systems were found to be in agreement with the observed distribution. The two main areas of disagreement with observed stellar properties were that the calculation produced a much higher ratio of brown dwarfs to stars than observed and there was a deficit of unequal-mass solar-type binaries.

However, the fact that a calculation only including gravity and fluid dynamics (without the more complicated physics of radiative transfer and magnetic fields) can reproduce many of the observed statistical properties of stellar systems implies that the origin of these properties is primarily due to dissipative gravitational dynamics and not significantly altered by additional processes. In many ways, this is surprising because, for example, magnetic fields are thought to be highly effective at transporting angular momentum and radiative feedback is likely to inhibit fragmentation near existing protostars.

\section{Radiation hydrodynamical simulations}

Thus, the question moves on to what the role of additional physical processes is in the star-formation process. Bate (2009b) recently repeated the two cluster formation calculations of Bate et al. (2003) and Bate \& Bonnell (2005), this time including a realistic equation of state and radiative transfer in the flux-limited diffusion approximation. The calculations modelled gas to within $0.5 \mathrm{AU}$ of each protostar and the energy released down to such scales, but did not include the radiative feedback coming from the stars themselves or discs within 0.5 AU. Despite this, the inclusion of radiative feedback from the forming protostars back into the cloud had a huge effect on the fragmentation. Massive circumstellar discs, in particular, were found to be much hotter and more resistant to fragmentation than in the barotropic calculations. The result was that in both calculations, the numbers of objects formed were reduced by a factor of $\approx 4$ from the original calculations. This, in turn, led to fewer dynamical interactions between objects and fewer ejections. Since the ejected objects tend to end up as low-mass stars and brown dwarfs, the ratio of brown dwarfs to stars was reduced by the inclusion of radiative feedback, bringing the IMFs produced by the calculations into good agreement with observations.

In addition, as mentioned in the previous section, using a barotropic equation of state, the stellar IMF was found to scale with the mean Jeans mass of the clouds. However, with radiative transfer, the IMFs produced from the two clouds were found to be indistinguishable despite their differing mean Jeans masses. Thus, Bate (2009b) proposed that radiative feedback from protostars self-regulates the star-formation process and erases, or severely weakens, the dependence of the IMF on the mean thermal Jeans mass of the progenitor cloud. This may help to explain the observed invariance of the IMF.

Following these calculations, Offner et al. (2009) also performed radiation hydrodynamical calculations of the collapse of molecular clouds to form low-mass stars. Their calculations were performed using adaptive-mesh refinement while Bate used smoothedparticle hydrodynamics. Unlike Bate, Offner et al. included radiative feedback from the stellar objects themselves and their accretion luminosity, but they used much larger accretion radii of $\approx 130 \mathrm{AU}$. Despite their differences, both studies found that radiative 
feedback dramatically reduced the number of objects formed relative to calculations performed using a barotropic equation of state and, therefore, that even in the case of low-mass star formation, it is crucial to include the effects of radiative feedback.

\section{Magnetohydrodynamical simulations}

Magnetic fields have long been recognised as a potentially important physical process in star formation. However, while their role in some aspects of star formation is clearly crucial (e.g., protostellar jets), their role in star cluster formation is less clear. Recent ideal magnetohydrodynamical (MHD) calculations have confirmed that magnetic fields can play a crucial role in protostellar-disc formation (Price \& Bate 2007; Hennebelle \& Fromang 2008) and the fragmentation of isolated molecular-cloud cores to form binary and multiple systems (Price \& Bate 2007; Hennebelle \& Teyssier 2008). However, their role in cluster formation has only recently begun to be investigated numerically.

Price \& Bate (2008) recently repeated the cluster-formation calculation of Bate et al. (2003), this time including magnetic fields of varying strengths using the ideal MHD approximation. While even at low strengths, the magnetic field was found to significantly influence the star-formation process, for magnetic fields where the ratio of gas to magnetic pressure was less than unity (i.e., plasma $\beta<1$ ) the results were substantially different to the hydrodynamic case. Anisotropic turbulent motions and column-density striations aligned with the magnetic-field lines were found, both of which have recently been observed in the Taurus molecular cloud (Goldsmith et al. 2008). In addition, largescale magnetically supported voids were produced. The additional large-scale support provided by the magnetic field strongly suppressed collapse in the clouds, leading to up to a $75 \%$ reduction in the amount of mass converted into stars over the course of the calculations. This led to a more quiescent mode of star formation and Price \& Bate found that the relative formation efficiency of brown dwarfs was lower in the strongly magnetized runs because of a reduction in the importance of protostellar ejections.

\section{Radiation magnetohydrodynamical simulations}

Most recently, Price \& Bate (2009) combined their treatments of magnetic fields and radation hydrodynamics to perform the first radiation magnetohydrodynamical calculations of star cluster formation. Again, the calculations were of small $50 \mathrm{M}_{\odot}$ molecular clouds as modelled by Bate et al. (2003), Bate (2009b) and Price \& Bate (2008).

The effects of radiative feedback and magnetic fields, found separately in the earlier studies, were combined in these calculations. The main effect of radiative feedback was to inhibit fragmentation on small scales, while the main effect of magnetic fields was to provide support to the low-density gas on large scales, decreasing the star-formation rate. With strong magnetic fields and radiative feedback, the net result was an inefficient star-formation process with a star-formation rate of $\approx 10 \%$ per free-fall time. This is much less than the rates found without magnetic fields (typically $\approx 50 \%$ per free-fall time) and approaches the observed rate of $\sim 3-6 \%$ (Evans et al. 2009).

\section{The future}

In summary, calculations of star cluster formation that only take into account gravity and compressible fluid dynamics can reproduce many of the observed properties of stellar systems, including many binary properties. They also produce stellar mass distributions in qualitative agreement with the observed IMF. Such properties do not appear to depend 
sensitively on the properties of the molecular-cloud turbulence as long as the turbulence is decaying or driven on large scales (as seems to be observed; Brunt et al. 2009). This is due to the nature of competitive accretion and dynamical interactions between protostars, which determine the spectrum of stellar masses in such calculations. These are local processes that have little memory of the large-scale initial conditions.

However, it is clear that both radiative transfer and magnetic fields need to be incorporated into future calculations of star cluster formation. Radiative feedback, even from low-mass protostars, is crucial to obtain quantitative agreement with the observed IMF and may help to explain why star formation does not depend sensitively on other initial conditions in molecular clouds (such as their density and temperature). Dynamically important magnetic fields seem to be required to explain the low rate of star formation and many of the structures observed in molecular clouds.

The challenges for the future are clear: to incorporate the effects of radiative transfer and magnetic fields into larger calculations that are capable of making precise predictions of stellar properties. Ideally, the methods for including radiative transfer and magnetic fields should also be improved. For radiative transfer, this means going beyond the flux-limited diffusion approximation, while for magnetic fields the nonideal effects of ambipolar diffusion, Hall conduction and resistivity should be included.

\section{References}

Bate, M. R. 2005, MNRAS, 363, 363

Bate, M. R. 2009a, MNRAS, 392, 590

Bate, M. R. 2009b, MNRAS, 392, 1363

Bate, M. R. 2009c, MNRAS, 397, 232

Bate, M. R. \& Bonnell, I. A. 2005, MNRAS, 356, 1201

Bate, M. R., Bonnell, I. A., \& Bromm, V. 2002a, MNRAS (Letters), 332, L65

Bate, M. R., Bonnell, I. A., \& Bromm, V. 2002b, MNRAS, 336, 705

Bate, M. R., Bonnell, I. A., \& Bromm, V. 2003, MNRAS, 339, 577

Bonnell, I. A., Bate, M. R., Clarke, C. J., \& Pringle, J. E. 1997, MNRAS, 285, 201

Bonnell, I. A., Bate, M. R., \& Vine, S. G. 2003, MNRAS, 343, 413

Bonnell, I. A., Clarke, C. J., \& Bate, M. R. 2006, MNRAS, 368, 1296

Brunt, C. M., Heyer, M. H., \& Mac Low, M.-M. 2009, MNRAS, 504, 883

Evans, N. J., et al. 2009, ApJS, 181, 321

Goldsmith, P. F., Heyer, M., Narayanan, G., Snell, R., Li, D., \& Brunt, C. 2008, ApJ, 680, 428

Hennebelle, P. \& Fromang, S. 2008, $A \mathscr{E} A$ A, 477, 9

Hennebelle, P. \& Teyssier, R. 2008, A\&A, 477, 25

Jappsen, A.-K., Klessen, R. S., Larson, R. B., Li, Y., \& Mac Low, M.-M. 2005, A $\& A, 435,611$

Klessen, R. S. 2001, ApJ, 550, 77

Klessen, R. S. \& Burkert, A. 2000, ApJS, 128, 287

Klessen, R. S. \& Burkert, A. 2001b, ApJ, 549, 386

Klessen, R. S., Burkert, A., \& Bate, M. R. 1998, ApJ (Letters), 501, L205

Krumholz, M. R., McKee, C. F., \& Klein, R.I. 2004, ApJ, 611, 399

Larson, R. B. 1981, MNRAS, 194, 809

Offner, S. S. R., Klein, R. I., \& McKee, C. F. 2008, ApJ, 686, 1174

Offner, S. S. R., Klein, R. I., McKee, C. F., \& Krumholz, M. R. 2009, ApJ, 703, 131

Price, D. J. \& Bate, M. R. 2007, MNRAS, 377, 77

Price, D. J. \& Bate, M. R. 2008, MNRAS, 385, 1820

Price, D. J. \& Bate, M. R. 2009, MNRAS, 398, 33 